\title{
The Energy Loss of $\alpha$-Particles in Ferromagnetic Nickel at Phase Transition
}

\author{
U. V. Khadke \\ Department of Physics, Bheemanna Khandre Institute of Technology, Bhalki 585328, India \\ Correspondence should be addressed to U. V. Khadke; khadke@email.com
}

Received 27 April 2016; Accepted 31 July 2016

Academic Editor: Stefano Bellucci

Copyright ( 2016 U. V. Khadke. This is an open access article distributed under the Creative Commons Attribution License, which permits unrestricted use, distribution, and reproduction in any medium, provided the original work is properly cited.

The energy loss (EL) of $5.486 \mathrm{MeV} \alpha$-particles in ferromagnetic nickel foils was measured at different temperatures of the foils. The temperature of the foils was controlled and recorded using a PC-based temperature controller with an accuracy of $\pm 0.01^{\circ} \mathrm{C}$. It is observed that the energy loss in ferromagnetic nickel increases by $2-5 \%$ as the material goes from ferromagnetic to paramagnetic state. Thus our results show that the phase transition in ferromagnetic materials affects energy loss.

\section{Introduction}

The study on the phenomenon of penetration of atomic particles through matter is extremely important as they provide a valuable tool for various possible applications such as radiation damage, radiation shielding, and radiation detector development. The reduction in energy loss of the projectile due to dielectric polarisation of the medium is normally referred to as "density effect" as this can be appreciable only in a dense medium. Many experimental results obtained with different projectiles agree well with the theory of Fermi and the detailed calculations of Sternheimer.

The energy loss (EL) of high energy electrons in ferroelectric target is anomalously high at ferroelectric transition temperature $T_{c}[1,2]$. These results indicate that there is a new mode of EL in ferroelectric materials, which is different from any of the known mechanisms of EL.

Ferromagnetic materials are analogous to ferroelectrics and exhibit a long-range ordering phenomenon at the atomic level, which causes the unpaired electron spins to line up parallel with each other in a ferromagnetic domain. Hence, as in the case of ferroelectrics, energy loss of charged particles in ferromagnetism may be affected by the ferromagnetic phase transition. The EL of $\alpha$-particles in iron and gadolinium were measured [3] and it was observed that the EL in paramagnetic state was higher by about $0.2 \%-1.5 \%$ than in ferromagnetic state. Ferromagnetism is much better understood phenomenon compared to the ferroelectricity. Hence in this paper we report the results obtained on the EL of $5.486 \mathrm{MeV} \alpha$-particles in ferromagnetic nickel.

\section{Experimental Set-Up}

The energy loss of $5.486 \mathrm{MeV} \alpha$-particles $\left(\mathrm{Am}^{241}\right)$ in ferromagnetic $\mathrm{Ni}(\mathrm{Ni}-58)$ of thickness $3 \mathrm{mg} / \mathrm{cm}^{2}$ (from Good fellow USA) was measured in transmission geometry. The two foils of uniform thickness were stacked in staircase configuration leaving a small gap at upper side of the opening in sample holder $(\mathrm{SH})$. So when a beam of $\alpha$-particles is incident on the sample in $\mathrm{SH}$ it is divided into three parts. First one goes through the opening, second one through one layer of film, and third one through two layers of film. The Solid State Surface Barrier Detector of $2 \mathrm{~mm}$ thick used to record the energy spectrum shows the incident beam, a beam transmitted through one layer of film, and a beam transmitted through two layers of film, separated from each other by a distance which depends on the thickness of each layer of film. This ensured measurement of EL of $\alpha$-particles in nickel foils with an accuracy of $0.05 \%$ that was free from effects of drifts, if any, in performance of the electronics modules used. From the recorded spectrum it is then possible to find EL of $\alpha$-particles in one layer, two layers, and so forth of the film. A Lakeshore model DRC-93CA temperature controller with IEEE-488 interface supplied the current to the heating element and also measured the temperature of the sample 


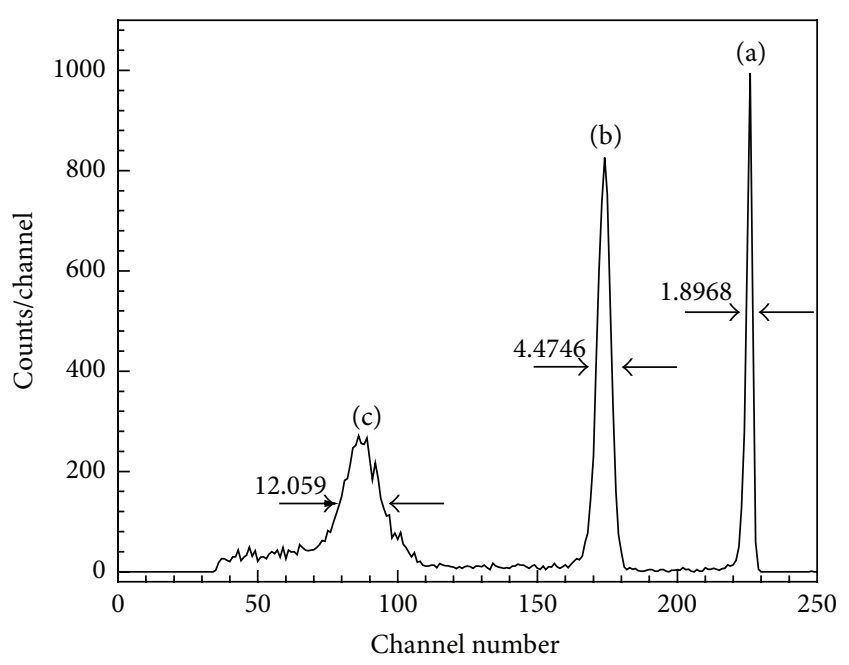

Figure 1: $\alpha$-particle spectrum with $\mathrm{Ni}$ foil at room temperature: (a) Incident beam, (b) beam transmitted through $3 \mathrm{mg} / \mathrm{cm}^{2}$ thick sample, and (c) beam transmitted through $6 \mathrm{mg} / \mathrm{cm}^{2}$ thick sample.

and detector by RTD sensors with an accuracy of $\pm 0.1^{\circ} \mathrm{C}$ over longer duration [4].

\section{Result and Discussion}

A typical spectrum recorded with $\mathrm{Ni}$ foil at room temperature is shown in Figure 1. The $\alpha$-particle spectrum consisted of three peaks, one sharp peak corresponding to the incident beam and the other two broad asymmetric peaks to the two transmitted beams. It is observed that the broadening of the peak increases with increase in foil thickness is due to straggling effect. The centroid of the incident and transmitted peak channel is obtained by the least-squares fitted to a Gaussian curve. However, for the third peak, only the $80 \%$ of entire peak is fitted to the Gaussian as it has broadening effect. From the incident peak, the calibration factor is $24.289 \mathrm{keV} /$ channel and the resolution is $0.84 \%$. Compared with SRIM value, the measured EL is lower by $8.67 \%$ in the first foil and higher by $9.94 \%$ in the second film.

All the spectra recorded at different temperature were processed in this manner. The variation of energy of transmitted beam energy with the temperature of the $3 \mathrm{mg} / \mathrm{cm}^{2}$ and $6 \mathrm{mg} / \mathrm{cm}^{2}$ foils is shown in Figures 2 and 3, respectively. The corresponding EL in the two foils is shown in Figure 4.

The incident beam energy was between $5.483 \mathrm{MeV}$ and $5.487 \mathrm{MeV}$ throughout the experiment. The transmitted beam energy was measured with accuracies of $1 \mathrm{keV}(0.02 \%)$ and $3 \mathrm{keV}(0.14 \%)$ in the first and second film, respectively. The EL in the two films was measured with accuracies of $2 \mathrm{keV}$ $(0.16 \%)$ and $4 \mathrm{keV}(0.12 \%)$. Hence experimental EL values are accurate and reliable.

From Figure 4 we observe following variations in EL with temperature of the foil. For the $3 \mathrm{mg} / \mathrm{cm}^{2}$ foil EL lies between $1.260 \mathrm{MeV}$ and $1.275 \mathrm{MeV}$ over the entire temperature range from $50^{\circ} \mathrm{C}$ to $340^{\circ} \mathrm{C}$ in the ferromagnetic region. As the ferromagnetic phase transition temperature of $353.85^{\circ} \mathrm{C}$ is

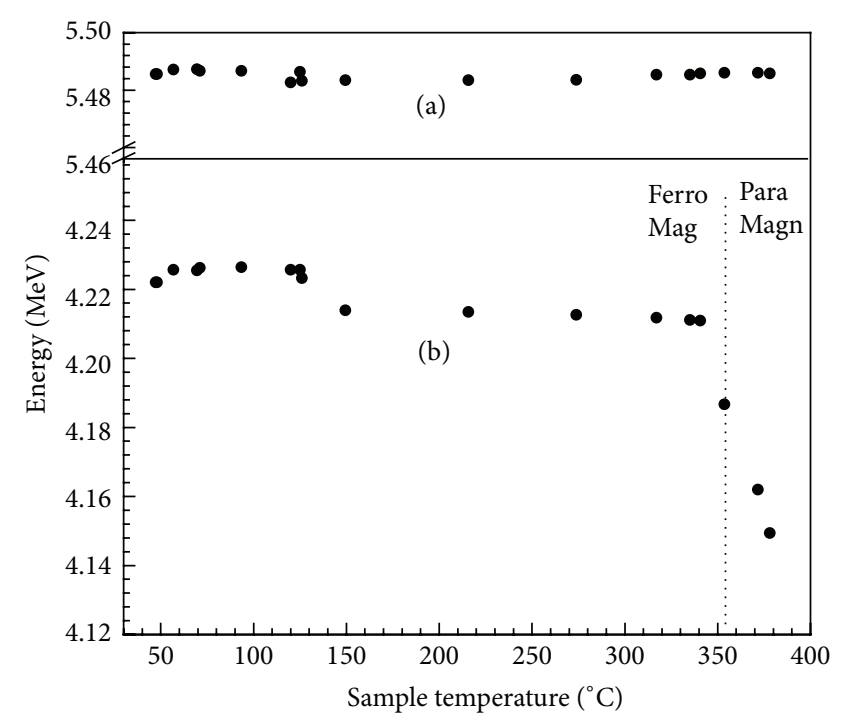

FIgURE 2: Variation of the $5.486 \mathrm{MeV} \alpha$-particle energy with temperature of $3 \mathrm{mg} / \mathrm{cm}^{2}$ thick nickel foil: (a) incident beam and (b) transmitted beam.

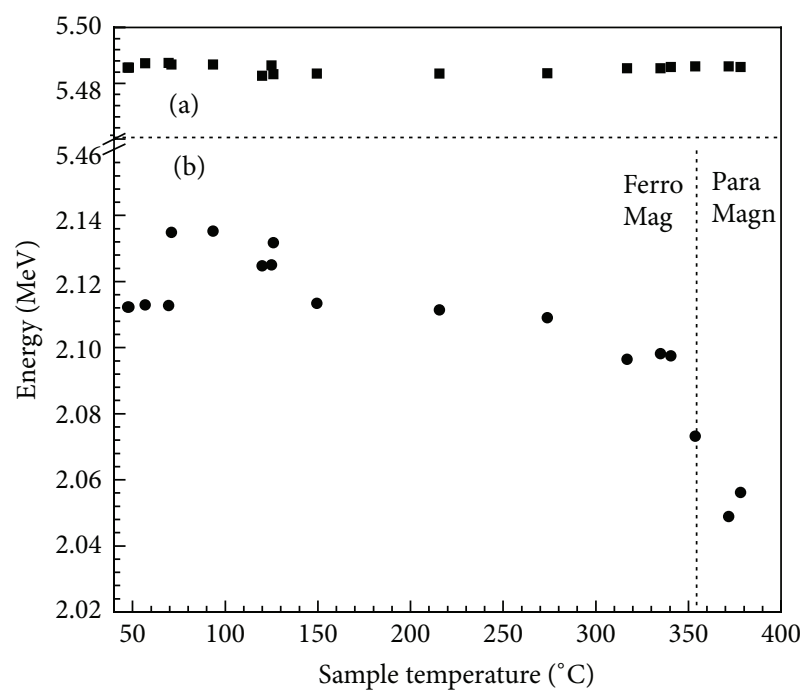

FIGURE 3: Variation of the $5.486 \mathrm{MeV} \alpha$-particle energy with temperature of $6 \mathrm{mg} / \mathrm{cm}^{2}$ thick nickel foil: (a) incident beam and (b) transmitted beam.

approached EL increases sharply and it has a value of $1.336 \mathrm{MeV}$ at $380^{\circ} \mathrm{C}$ in the paramagnetic phase. Thus EL increases by $65 \mathrm{keV}$, that is, by $5.1 \%$ as the material goes from ferro- to paramagnetic state. However, for the $6 \mathrm{mg} / \mathrm{cm}^{2}$ foil EL lies between $3.358 \mathrm{MeV}$ and $3.375 \mathrm{MeV}$ over the entire temperature range from $50^{\circ} \mathrm{C}$ to $340^{\circ} \mathrm{C}$ in the ferromagnetic region. As the ferromagnetic phase transition temperature of $353.85^{\circ} \mathrm{C}$ is approached EL increases sharply and it has a value of $3.430 \mathrm{MeV}$ at $380^{\circ} \mathrm{C}$ in the paramagnetic phase. Thus EL increases by $64 \mathrm{keV}$, that is, by $1.9 \%$ as the material goes from ferro- to paramagnetic state. 


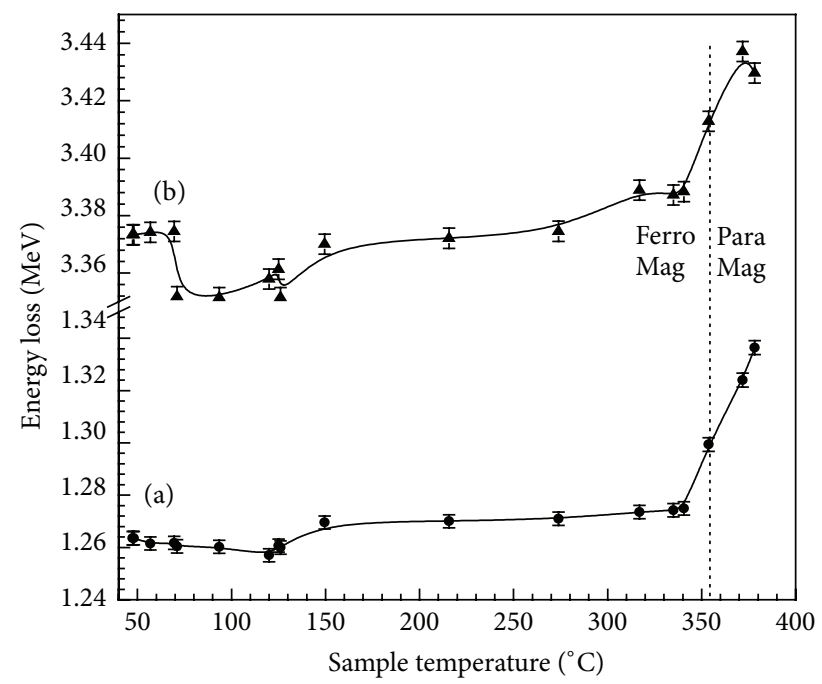

Figure 4: Variation of energy loss of $5.486 \mathrm{MeV} \alpha$-particles in $\mathrm{Ni}$ foil: (a) $3 \mathrm{mg} / \mathrm{cm}^{2}$ thick sample and (b) $6 \mathrm{mg} / \mathrm{cm}^{2}$ thick sample.

The observed enhancement of EL around the phase transition temperature, compared to its value in the ferromagnetic phase, is similar to the results for EL [1-3].

The observed results cannot be attributed to changes in mean excitation potential as the required change in mean excitation potential to give such change in EL would be too large to be realized by phase change [3]. The results are indicative of a new mode of energy loss which is not included in the conventional theory of stopping power of a medium. The possible new mechanism may be as follows: (1) the magnetic fields associated with moving particles are weaker by a factor of 1/137 compared to the electric field; (2) the field is further small because the velocity of $\alpha$-particles of $5-6 \mathrm{MeV}$ is very small. But there is a positive aspect also: the magnetic dipole moment in a ferromagnet is associated with the individual atom. Thus the magnetic field of the moving particle has to interact with individual atomic dipoles and could therefore lead to change in EL. It may not be necessary for the magnetic field to interact with the magnetic domain to account for critical energy loss near $T_{c}$. Nevertheless, role of collective effects in ferromagnetic material on the EL is not entirely ruled out and the results emphasize need for further studies in this area. Since there is an enhancement in EL the phenomenon could have an application in radiation shielding [5].

\section{Conclusion}

The energy loss of $5.486 \mathrm{MeV} \alpha$-particles in ferromagnetic nickel shows that there is discontinuity in energy loss at the ferromagnetic transition. Our results show that the phase transition in ferromagnetic material affects energy loss.

\section{Competing Interests}

The author declares that there are no competing interests regarding the publication of this paper.

\section{Acknowledgments}

The author is grateful to the Director, Inter University Accelerator Centre (IUAC) New Delhi, for having provided the energy spectrometer setup for the measurement.

\section{References}

[1] M. T. Lagare and N. Umakantha, "Anomalous energy loss of electrons in ferroelectrics at the curie temperature," Physical Review Letters, vol. 49, no. 19, pp. 1431-1433, 1982.

[2] P. Ayyub, M. S. Multani, M. R. Srinivasan et al., "Enhancement of energy loss of fast electrons in a ferroelectric medium near the Curie temperature," Physical Review B, vol. 32, no. 5, pp. 2835-2840, 1985.

[3] F. Nickel, H. Folger, H. Geissel et al., "Energy-loss differences of alpha particles in ferromagnetic and paramagnetic solids," Nuclear Instruments and Methods in Physics B, vol. 90, no. 1-4, pp. 20-23, 1994.

[4] K. Udaykumar, B. R. Kerur, S. M. Hangodimath et al., "The effect of temperature on the behavior of semiconductor silicon surface barrier detectors," Radiation Measurements, vol. 36, no. 1-6, pp. 625-628, 2003.

[5] M. T. Lagare and N. Umakantha, "Potential of ferroelectrics as radiation shields," Nature, vol. 332, no. 6166, p. 689, 1988. 

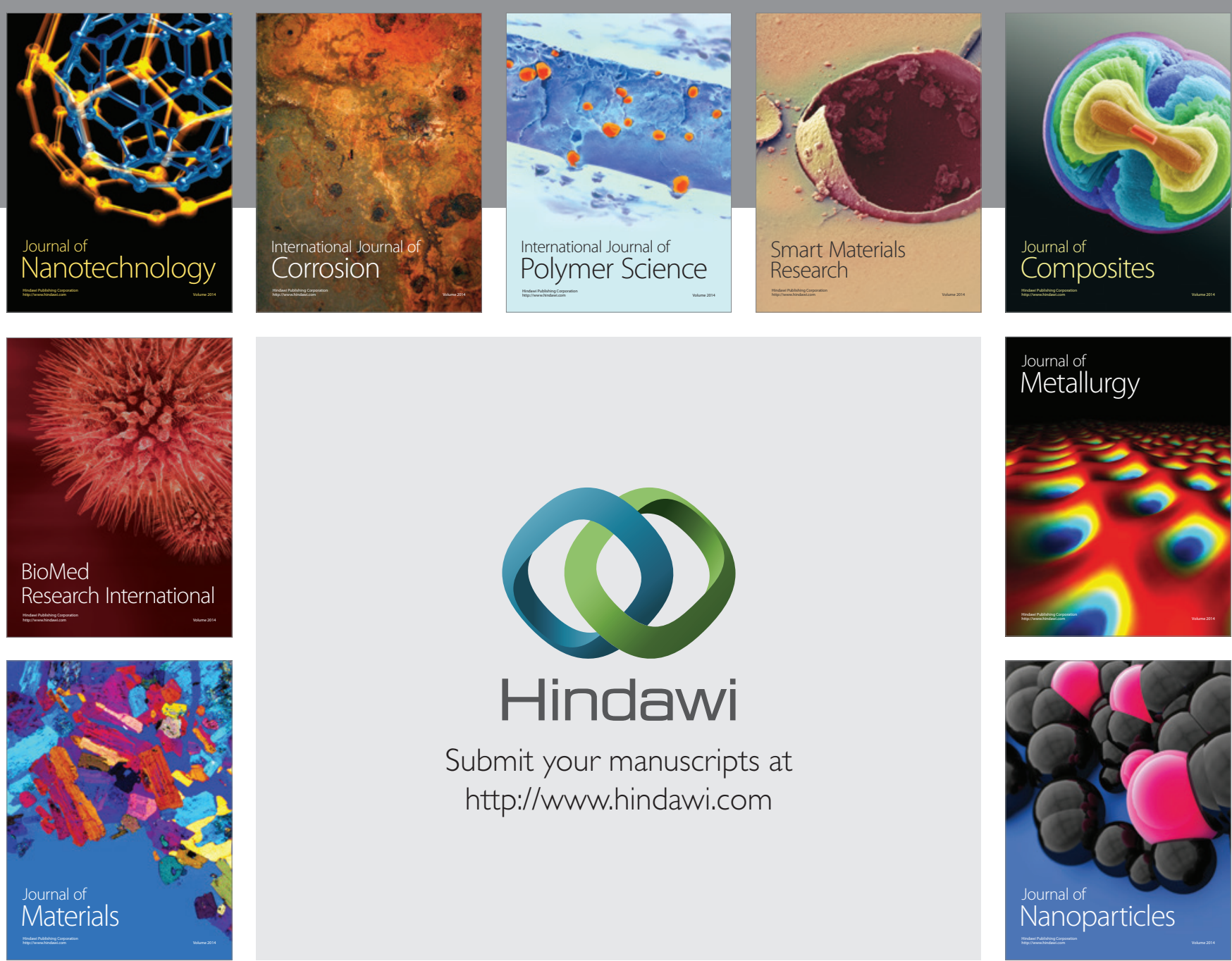

\section{Hindawi}

Submit your manuscripts at

http://www.hindawi.com

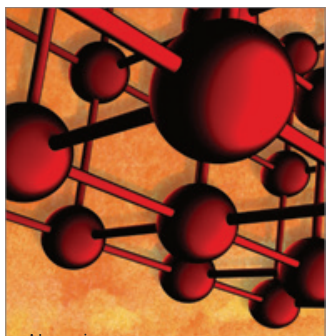

Materials Science and Engineering
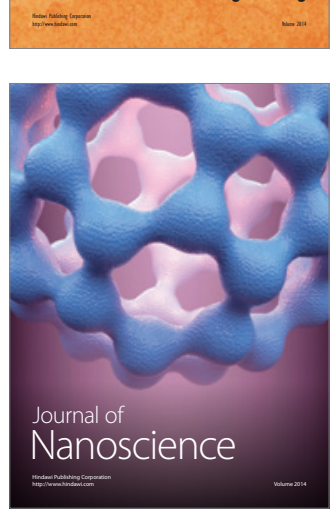
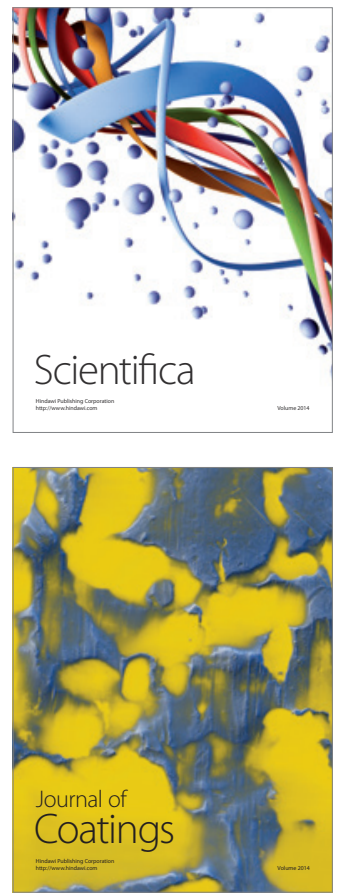
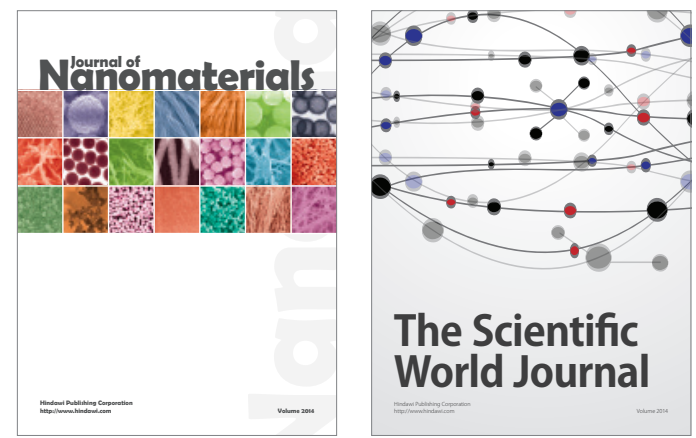

The Scientific World Journal
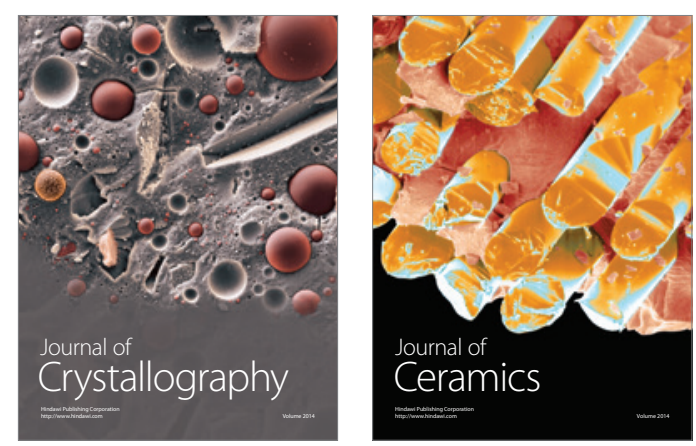
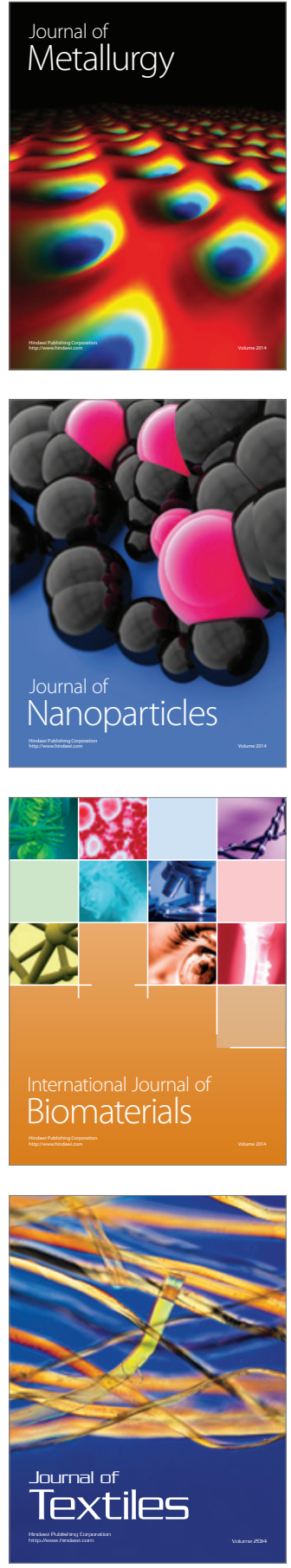